\title{
Understanding heart failure with preserved ejection fraction: where are we today?
}

\author{
L. van Heerebeek ${ }^{1,2} \cdot$ W.J. Paulus ${ }^{1}$
}

Published online: 24 February 2016

(C) The Author(s) 2016. This article is published with open access at Springerlink.com

\begin{abstract}
Heart failure with preserved ejection fraction (HFpEF) represents a complex and heterogeneous clinical syndrome, which is increasingly prevalent and associated with poor outcome. In contrast to heart failure with reduced ejection fraction ( $\mathrm{HFrEF}$ ), modern heart failure pharmacotherapy did not improve outcome in HFpEF, which was attributed to incomplete understanding of HFpEF pathophysiology, patient heterogeneity and lack of insight into primary pathophysiological processes. HFpEF patients are frequently elderly females and patients demonstrate a high prevalence of non-cardiac comorbidities, which independently adversely affect myocardial structural and functional remodelling. Furthermore, although diastolic left ventricular dysfunction represents the dominant abnormality in HFpEF, numerous ancillary mechanisms are frequently present, which also negatively impact on cardiovascular reserve. Over the past decade, clinical and translational research has improved insight into HFpEF pathophysiology and the importance of comorbidities and patient heterogeneity. Recently, a new paradigm for HFpEF was proposed, which states that comorbidities drive myocardial dysfunction and remodelling in HFpEF through coronary microvascular inflammation. Regarding the conceptual framework of HFpEF treatment, emphasis may need to shift from a 'one fits all' strategy to an indi-
\end{abstract}

L. van Heerebeek

1.vanheerebeek@vumc.nl

1 Institute for Cardiovascular Research VU (ICaR-VU), VU University Medical Center Amsterdam, Amsterdam, The Netherlands

2 Department of Cardiology, Onze Lieve Vrouwe Gasthuis, Amsterdam, The Netherlands vidualised approach based on phenotypic patient characterisation and diagnostic and pathophysiological stratification of myocardial disease processes. This review will describe these novel insights from a pathophysiological standpoint.

Keywords Heart failure · Diastole · Inflammation · Endothelial dysfunction

\section{Introduction}

Heart failure with preserved ejection fraction (HFpEF) currently accounts for more than $50 \%$ of all heart failure patients, and its prevalence relative to heart failure with reduced ejection fraction (HFrEF) is rising at a rate of approximately $1 \%$ per year, while HFpEF patients have only slightly lower mortality rates than HFrEF patients [1]. By 2020 , the prevalence of HFpEF will exceed $8 \%$ of persons older than 65 years of age and the relative prevalences of HFpEF and HFrEF are predicted to be 69 and $31 \%$, turning HFpEF into the most common heart failure phenotype [1]. HFpEF is diagnosed in the presence of heart failure signs and/or symptoms, preserved systolic left ventricular (LV) function, with an LV ejection fraction (LVEF) $>50 \%$ and LV end-diastolic volume index $<97 \mathrm{ml} / \mathrm{m}^{2}$ with evidence of diastolic LV dysfunction [2]. In contrast to HFrEF, modern heart failure pharmacotherapy did not improve the prognosis in HFpEF and all large randomised HFpEF trials [3-7] have yielded neutral results. These neutral results of recent $\mathrm{HFpEF}$ trials were attributed to an incomplete understanding of HFpEF pathophysiology, suboptimal study designs, inadequate diagnostic criteria or statistical power, patient heterogeneity and poor matching of therapeutic mechanisms and primary pathophysiological processes [8]. In the past decade, clinical and translational research pro- 
vided exiting novel insights into HFpEF pathophysiology and the importance of comorbidities and patient heterogeneity, which could be of great interest for the design and interpretation of future trials as will be discussed in this review.

\section{Pathophysiology of diastolic LV dysfunction in HFpEF}

Under physiological conditions, LV pressure rapidly decreases after systole, allowing fast diastolic LV filling at maintained low filling pressures. Diastolic LV dysfunction in HFpEF is evident from slow LV relaxation and elevated diastolic LV stiffness, which increase diastolic filling pressures and limit cardiac performance at rest, during atrial pacing and exercise $[9,10]$. Insight into the pathophysiology of diastolic LV dysfunction in HFpEF has long been missing because of a lack of myocardial tissue obtained from patients with HFpEF. Over the past decade, several groups of investigators were able to obtain myocardial tissue from HFpEF patients revealing specific alterations in myocardial structure, function and intramyocardial signalling, which were relevant to the concentric LV remodelling and diastolic LV dysfunction characteristically observed in patients with HFpEF (Table 1; [11-18]). Structural alterations consisted of cardiomyocyte hypertrophy $[12,13]$ and varying degrees of myocardial interstitial fibrosis [11-13, 17, 18] and capillary rarefaction [18], whereas functional alterations included increased cardiomycyte stiffness [11-15]. The same studies also demonstrated abnormal intramyocardial signalling, which was evident from endothelial cells expressing adhesion molecules $[13,16]$, inflammatory cells secreting profibrotic transforming growth factor $\beta$ (TGF- $\beta$ ) [16] oxidative stress increasing nitrotyrosine content $[15,16]$ and downregulation of myocardial cyclic guanosine monophosphate (cGMP)-protein kinase G (PKG) signalling [15]. Myocardial cGMP-PKG signalling is crucial for normal cardiovascular physiology, inhibiting maladaptive hypertrophy and enhancing cardiomyocyte compliance through PKG-mediated

Table 1 Specific alterations in myocardial structure, function and intramyocardial signalling demonstrated in HFpEF patients

\begin{tabular}{lll}
\hline Structural alterations & $\begin{array}{l}\text { Functional } \\
\text { alterations }\end{array}$ & $\begin{array}{l}\text { Intramyocardial signal- } \\
\text { ling alterations }\end{array}$ \\
\hline $\begin{array}{l}\text { Cardiomyocyte } \\
\text { hypertrophy }\end{array}$ & $\begin{array}{l}\text { Increased cardio- } \\
\text { myocyte stiffness }\end{array}$ & $\begin{array}{l}\text { Endothelial cells } \\
\text { expressing adhesion } \\
\text { molecules }\end{array}$ \\
Interstitial fibrosis & $\begin{array}{l}\text { Impaired cardio- } \\
\text { myocyte relaxation }\end{array}$ & $\begin{array}{l}\text { Inflammatory cells } \\
\text { secreting TGF- } \beta \\
\text { Oxidative stress } \\
\text { increasing nitrotyrosine } \\
\text { capillary rarefaction }\end{array}$ \\
& & $\begin{array}{l}\text { Downregulation of } \\
\text { myocardial cGMP- } \\
\text { PKG signalling }\end{array}$ \\
\hline
\end{tabular}

phosphorylation of the sarcomeric protein titin $[19,20]$. Cardiomyocyte stiffness is mainly determined by the elastic sarcomeric protein titin, which functions as a bidirectional spring, responsible for early diastolic recoil and late diastolic distensibility [19]. Titin-based cardiomyocyte stiffness results from dynamic changes in expression of stiff (N2B) and compliant (N2BA) isoforms, from isoform phosphorylation status and from oxidative changes of the N2B segment [19]. Phosphorylation of titin by protein kinase A (PKA) and PKG increase its compliance, thereby acutely lowering cardiomyocyte stiffness (Fig. 1; [11-15]). Various studies, which procured endomyocardial tissue from patients with HFpEF, HFrEF and aortic stenosis, demonstrated significantly stiffer cardiomyocytes in HFpEF than in HFrEF and aortic stenosis patients [11-15]. This increased cardiomyocyte stiffness was related to increased titin $\mathrm{N} 2 \mathrm{~B}$ isoform expression, relative to HFrEF [12], and to reduced phosphorylation of titin [14]. Hypophosphorylation of titin resulted from lower myocardial PKG activity and reduced myocardial cGMP concentration in HFpEF compared with HFrEF and aortic stenosis [15]. The generation of the second messenger molecule cGMP results from activation of soluble guanylate cyclase (sGC) by nitric oxide (NO) and from activation of particulate GC (pGC) by natriuretic peptides (NPs) (Fig. 2; [20]). Once generated, cGMP activates PKG allowing PKG-mediated phosphorylation of a vast number of target proteins, exerting a wide range of downstream effects such as enhanced reuptake of calcium $(\mathrm{Ca} 2+)$ into the sarcoplasmic reticulum, inhibition of $\mathrm{Ca} 2+$ influx, suppression of hypertrophic signalling through inhibition of G-protein coupled receptors and the transient receptor potential canonical channel (TRPC), inhibition of ischaemia-reperfusion injury through phosphorylation of the ATP-sensitive potassium channel and stimulation of LV relaxation and LV distensibility by phosphorylation of troponin I (TnI) and the titin N2B segment (Fig. 2; [19, 20]). Downregulation of myocardial cGMP-PKG signalling in HFpEF is related to reduced myocardial brain-type NP (BNP) expression and increased microvascular inflammation and oxidative stress, which impair both the NP-cGMP and NO-cGMP axes (Fig. 2; [15]). Reduced myocardial BNP expression in HFpEF could have resulted from a number of factors, including concomitant obesity and insulin resistance, which lower myocardial BNP expression [21] and concentric LV remodelling/hypertrophy, which reduces both systolic and diastolic LV wall stress [22]. In addition, low myocardial BNP expression in HFpEF could also have resulted from increased expression of phosphodiesterase (PDE) type 9, which breaks down cGMP specifically generated through the NP-pGC axis [23]. Impaired NO-cGMP signalling could have resulted from the increased inflammation and oxidative stress observed in HFpEF, which was inferred from the high prevalence of comorbidities such as hypertension, obesity and diabetes mellitus type 2 (Fig. 2; [15]). 
Fig. 1 Cardiomyocyte cAMP and cGMP signalling pathways involved in myofilament regulation and titin-based stiffness. Stimulation of $\beta$-ARs activates Gs -ACmediated generation of cAMP, which stimulates PKA activity. cGMP is generated from activation of sGC by NO and from activation of pGC by NPs. cGMP stimulates PKG activity. Both effects through phosphorylation of TnI, and lower cardiomyocyte stiffness through phosphorylation of the titin N2B segment. Circled $\mathrm{P}$ 's indicate phosphorylatable sites. $A C$ adenylyl cyclase, $\beta A R$ beta-adrenergic receptor, $C A M P$ cyclic adenosine monophosphate, $G$ G-stimulatory protein, $N P R$ natriuretic peptide receptor, $P E V K$ unique sequence rich in proline, glutamic acid, valine and lysine PKA and PKG induce lusitropic

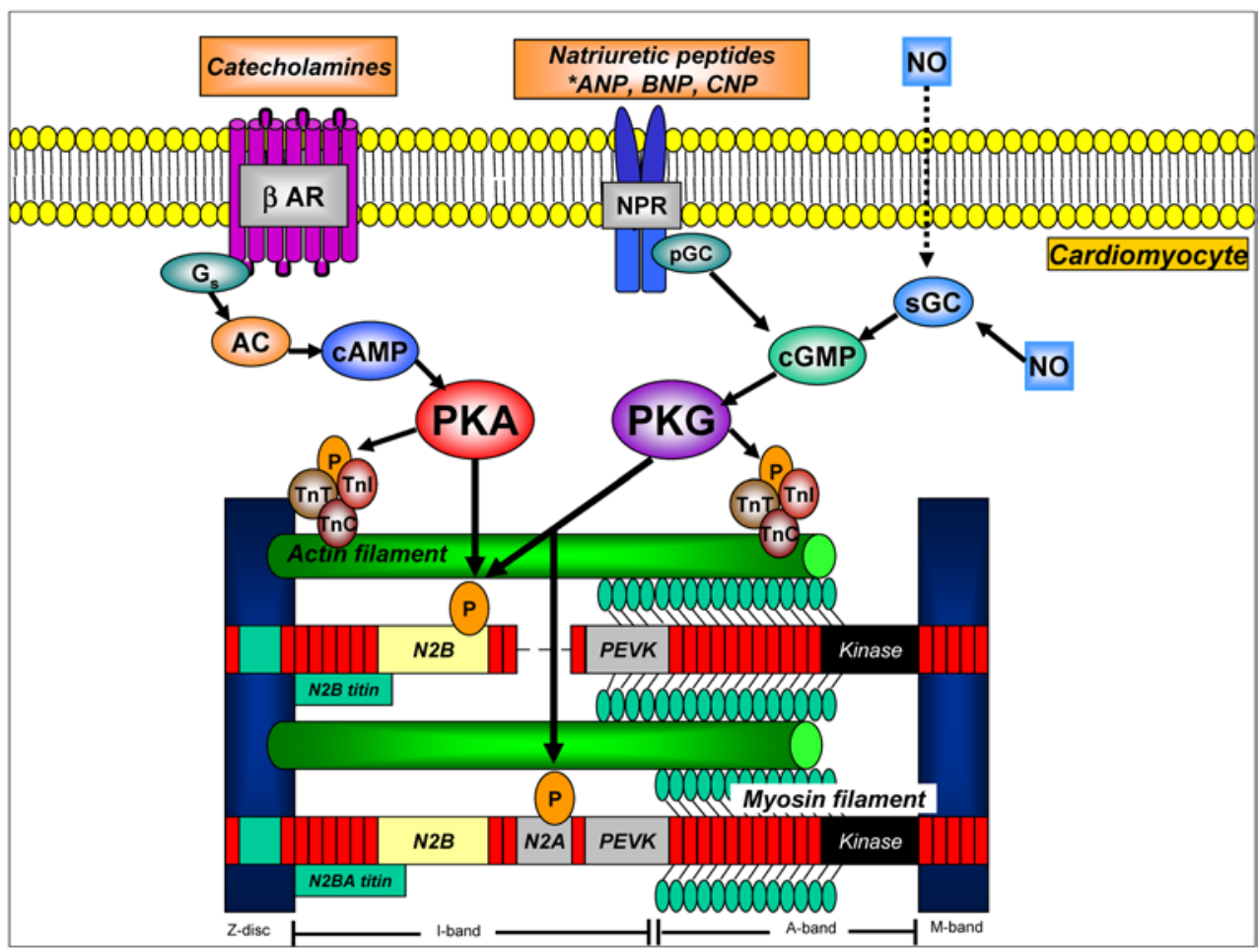

KEY MESSAGE HFpEF represents a heterogeneous clinical syndrome for which the conceptual framework of treatment may need to shift from a 'one fits all' strategy to an individualised approach based on phenotypic patient characterisation and diagnostic and pathophysiological stratification of myocardial disease processes. cGMP-PKG signalling in HFpEF. $P D E 5$ phosphodiesterase type 5, $P D E 9$ phosphodiesterase type 9 , $S R$ sarcoplasmic reticulum, $R G S 2 / 4$ regulator of G-protein signalling 2 and 4

\section{Comorbidities in HFpEF}

HFpEF patients are generally older, more often female and have a high prevalence of cardiovascular and non-cardiovascular comorbidities, such as obesity, metabolic syndrome, diabetes mellitus type 2, salt-sensitive hypertension, atrial fibrillation (AF), chronic obstructive pulmonary disease, anaemia and renal dysfunction [24-26]. Systemic inflammation and endothelial dysfunction are important hallmarks of these comorbidities and are also importantly involved in
HFpEF pathophysiology [16, 27]. Myocardial inflammation was shown to contribute to extracellular matrix changes in HFpEF and both myocardial collagen and the amount of inflammatory cells correlated with diastolic LV dysfunction [16]. Endothelial dysfunction is highly prevalent in HFpEF and is related to reduced exercise capacity and worse outcome [27]. Recently, a new paradigm of HFpEF was suggested, which attributes an important role of comorbidities for myocardial dysfunction and remodelling in $\mathrm{HFpEF}$ (Fig. 3; [28]). 


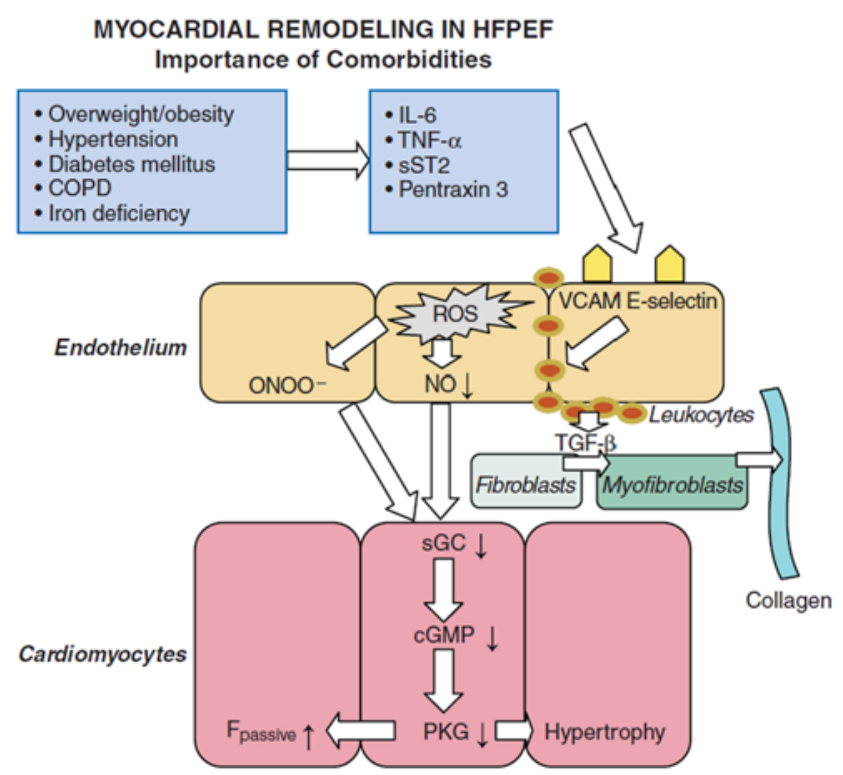

Fig. 3 Comorbidities drive myocardial dysfunction and remodelling in HFPEF. IL-6 interleukin-6; $s S T 2$ soluble ST2; $T N F-\alpha$ tumour necrosis factor alfa; $V C A M$ vascular cell adhesion molecule. Modified with permission from [28]

\section{The new paradigm of HFpEF}

The new HFpEF paradigm proposes that comorbidities drive structural and functional remodelling in $\mathrm{HFpEF}$ through systemic endothelial inflammation [28]. Because of this proinflammatory state, coronary microvascular endothelial cells produce reactive oxygen species, which limits NO bioavailability for adjacent cardiomyocytes. Impaired NO bioavailability results in downregulation of sGC-mediated cGMP-PKG signalling, which augments cardiomyocyte stiffness through hypophosphorylation of titin and increases cardiomyocyte hypertrophy because of impaired PKG-mediated antihypertrophic activity [28]. Furthermore, coronary microvascular endothelial inflammation favours subendothelial migration of leukocytes, which stimulates myofibroblast formation and interstitial collagen deposition. Both increased cardiomyocyte stiffness and interstitial fibrosis induce diastolic LV dysfunction [28].

Microvascular endothelial inflammation is also associated with myocardial capillary rarefaction [29], which was recently demonstrated in HFpEF myocardium [18].

\section{Myocardial capillary rarefaction}

A recent autopsy study demonstrated reduced myocardial capillary density (MCD) in HFpEF patients regardless of the severity of epicardial coronary disease, while the severity of myocardial fibrosis was inversely associated with MCD [18]. Both fibrosis and MCD were similar in those with or without hypertension, suggesting that comorbidities other than hypertension may perpetuate these alterations. Indeed, diabetes mellitus [30] and obesity [30,31] are known to be associated with MCD. In a recent study performing histological analysis of non-ischaemic myocardium from 57 patients undergoing coronary artery bypass graft surgery, obese patients had lower MCD [31]. Increased body mass index (BMI) was associated with higher pulmonary capillary wedge pressure (PCWP) and lower MCD was associated with both BMI and increased PCWP [31]. Myocardial capillary rarefaction in HFpEF may contribute to decreased maximal myocardial blood flow, impaired oxygen delivery and insufficient metabolic efficiency, which contribute to diastolic LV dysfunction and a higher risk of heart failure in obese individuals [31].

\section{Heterogeneity in HFpEF}

HFpEF is difficult to define as illustrated by various diagnostic classifications and inclusion criteria of clinical trials [8]. These factors contributed to heterogeneity of HFpEF patients recruited into trials and registries. Previously, no consensus was present on the optimal LVEF cut-off value and different cut-offs have been used across classifications and trials ranging from LVEF $\geq 40 \%$ to $>50 \%$ [8]. Although diastolic LV dysfunction represents the dominant abnormality in HFpEF, ancillary mechanisms may also contribute, such as systolic LV dysfunction [32, 33], ventricular-vascular stiffening [34], impaired systemic vasodilatory reserve [35], chronotropic incompetence [33, 35], pulmonary hypertension [36] and right ventricular (RV) dysfunction (Fig. 4; [37, 38]).

\section{Systolic dysfunction}

Although ejection fraction is preserved, both chamber and myocardial contractility were subtly but significantly depressed in HFpEF, compared with hypertensive and healthy controls [32]. The extent of myocardial contractile dysfunction in HFpEF related to increased mortality, suggesting that it may be a mediator or marker of more severe disease [32]. Even mild limitations in basal contractility may become more problematic with exercise stress $[32,33]$. During exercise, compared with controls, HFpEF patients demonstrated reductions in peak maximal oxygen uptake, chronotropic competence and relative increment in stroke volume and cardiac output [33]. Time to peak diastolic filling increased in HFpEF patients, while it decreased in the control group [33].

\section{Ventricular-vascular stiffening}

Maintaining low ventricular and arterial elastance allows a dynamic range of volume transfer to be achieved during ejection with minimal change in pressure. Ventricular and 


\title{
Advertisement placed here.
}

\author{
CSS Bohn

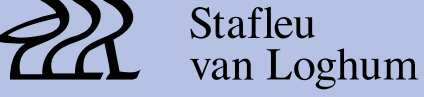 \\ Springer Media
}

Houten 2015 


\title{
Advertisement placed here.
}

\author{
CSS Bohn

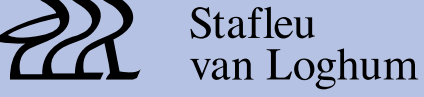 \\ Springer Media
}

Houten 2015 
Fig. 4 HFpEF represents a heterogeneous syndrome, characterised by multiple cardiovascular and non-cardiovascular comorbidities

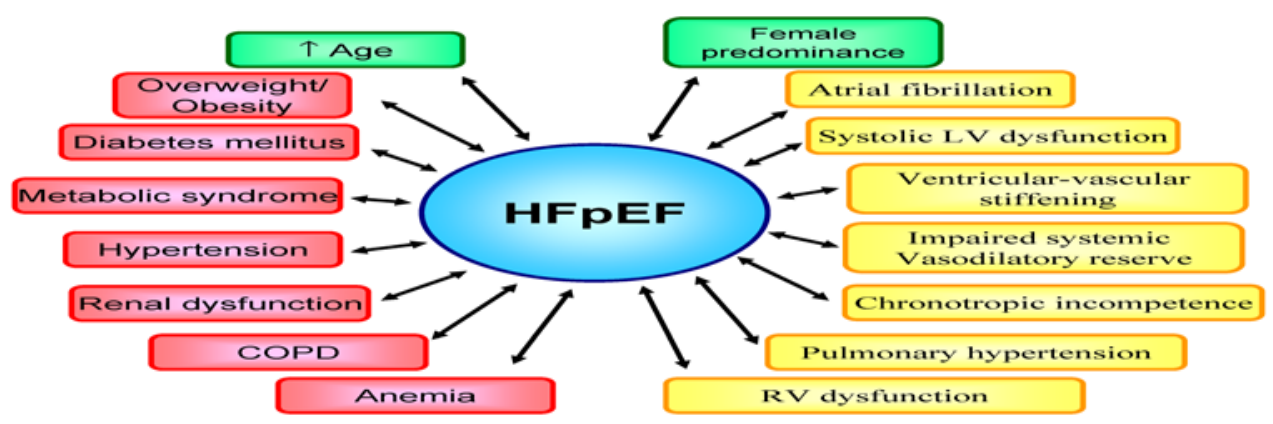

vascular stiffening increase with ageing, hypertension and diabetes and are abnormally elevated in HFpEF patients $[34,39]$. Combined ventricular-arterial stiffening elevates blood pressure lability, amplifying blood pressure changes for any alteration in preload or afterload. Furthermore, combined ventricular-arterial stiffening may also compromise endothelial-dependent vasorelaxation [39].

\section{Impaired systemic vasodilatory reserve}

Patients with HFpEF display attenuated exercise-mediated reductions in mean vascular resistance and arterial elastance, coupled with abnormalities in endothelial function and dynamic ventricular-arterial coupling compared with hypertensive subjects and controls [35]. The extent of impaired flow-mediated vasodilation is related to exercise intolerance [35]. The healthy endothelium has antiproliferative and anti-inflammatory actions and regulates vascular tone by balancing production of vasodilators and vasoconstrictors in response to a variety of stimuli [40]. In a prospective cohort study, endothelial dysfunction was highly prevalent in HFpEF patients and independently correlated with future cardiovascular events [27].

\section{Chronotropic incompetence}

Chronotropic reserve is depressed in $\mathrm{HFpEF}$ [33, 35], which could be related to downstream deficits in $\beta$-adrenergic stimulation, because the increase in plasma catecholamines with exercise is similar in HFpEF and healthy controls [41]. Autonomic dysfunction may contribute to chronotropic incompetence, as baroreflex sensitivity is reduced and heart rate recovery impaired in HFpEF [41].

\section{Pulmonary hypertension}

In a community-based study, the prevalence of pulmonary hypertension, defined as pulmonary artery systolic pressure (PASP) $>35 \mathrm{mmHg}$, amounted to $83 \%$ with a median PASP of $48 \mathrm{mmHg}$ in a group of $244 \mathrm{HFpEF}$ patients. In this study, PASP was significantly higher in HFpEF patients than in hypertensives without heart failure, whereas PASP strongly predicted mortality in HFpEF [36]. Chronic elevation of LV filling pressures induces LA remodelling and dysfunction, mixed pulmonary hypertension and ultimately, right ventricular (RV) remodelling and dysfunction [38].

\section{Right ventricular dysfunction}

In an invasive study, right and left heart filling pressures, pulmonary artery pressures and right-sided chamber dimensions were higher in HFpEF compared with controls, while LV size and ejection fraction were similar. RV dysfunction was present in $33 \%$ of HFpEF patients and was caused by both RV contractile impairment and afterload mismatch from pulmonary hypertension [37]. RV dysfunction was also associated with symptom severity and greater comorbidity burden [37]. In a prospective study, approximately one-third of HFpEF patients had evidence of RV dysfunction and both reduced LV compliance and RV dysfunction and remodelling were the strongest pathophysiological predictors of adverse outcomes [38].

\section{Phenotyping of HFpEF patients}

The underlying phenotypic heterogeneity is likely far greater in HFpEF than in HFrEF and may be an important reason for the failure of HFpEF clinical trials [42]. For instance, HFpEF patients with pulmonary hypertension and RV systolic dysfunction responded favourably to the phosphodiesterase type 5 inhibitor sildenafil [43], whereas sildenafil exerted no benefit in HFpEF patients without concomitant RV dysfunction [6]. Recently, phenomapping analysis using statistical learning algorithms demonstrated that HFpEF patients recruited according to uniform diagnostic criteria could be divided into three main distinct subgroups, which differed markedly in clinical characteristics, cardiac structure and function, invasive haemodynamics and outcome, despite similar endsystolic and end-diastolic elastances on LV pressure-volume analysis [42]. Therefore, understanding the phenotypic heterogeneity of HFpEF, which includes the aetiological and pathophysiologic heterogeneity of the syndrome, may allow more targeted and successful HFpEF clinical trials. 


\section{Identifying the severity of myocardial dysfunction and remodelling in HFpEF}

Recent HFpEF trials demonstrated structural cardiac remodelling in many HFpEF patients including concentric LV remodelling and hypertrophy (59-77\%) and left atrial (LA) dilatation (59-66\%) [5, 7]. Recruitment of LA contractility during stress is impaired in HFpEF and may contribute to the transition from an asymptomatic state to $\mathrm{HFpEF}$, while LA size also predicts clinical outcome [44]. Furthermore, translational studies investigating myocardial tissue from HFpEF patients demonstrated varying degrees of cardiomyoycte hypertrophy, interstitial fibrosis and capillary rarefaction $[11,12,18]$, implying distinct and possibly evolutionary stages of myocardial disease progression. Although all large HFpEF trials addressing renin-angiotensin-aldosterone system (RAAS) inhibitors failed to reach statistical significance for the primary outcome, many of them reached borderline significance for a primary endpoint or statistical significance for secondary endpoints, subgroups or post-hoc analyses $[3-5,7,45$, 46]. Because of these findings, the involvement of RAAS in HFpEF appears more subtle than in HFrEF, probably requiring up-front identification of subgroups of HFpEF patients rather than a 'one fits all strategy', which has hitherto been applied in HFpEF in analogy to HFrEF. Such a stratification approach with identification of myocardial structural and functional abnormalities in individual HFpEF patients could be relevant for the determination of potential therapeutic responsiveness and selection of appropriate treatment strategies. For instance, the efficacy of RAAS inhibitors to improve adverse myocardial remodelling is likely very different in HFpEF patients with minor, modest or severe stages of myocardial hypertrophy, fibrosis and capillary rarefaction (Fig. 5; [12]). Indeed, RAAS inhibitors might be effective in HFpEF patients presenting at an early stage, whereas therapeutic efficacy may be lost in HFpEF patients presenting at advanced or final stages. AF is likely to emerge as an indicator of advanced disease in HFpEF. A recent subgroup analysis of HFpEF patients recruited in the RELAX trial indeed showed presence of $\mathrm{AF}$ to be indicative of longstanding HFpEF [47]. In this study, HFpEF patients with AF were older than those in sinus rhythm, but had similar symptom severity, comorbidities, and renal function. Despite comparable LV size and mass, AF was associated with worse systolic (lower EF, stroke volume, and cardiac index) and diastolic (shorter deceleration time and larger left atria) function compared with sinus rhythm. Patients with AF had higher PASP and increased NT-proBNP, aldosterone, endothelin-1, troponin I, and C-telopeptide for type I collagen levels, suggesting more severe neurohumoral activation, myocyte necrosis, and fibrosis [47]. Multi-biomarker assessment and cardiac imaging modalities could represent promising tools for diagnostic stratification to identify the underlying stage of myocardial disease in the individual HFpEF patient.

\section{Identifying stage of myocardial disease}

\section{Biomarkers}

Currently, only NPs are routinely used for diagnosis and risk stratification in HFpEF patients. Because HFpEF is characterised by inflammation, oxidative stress, endothelial dysfunction, alterations in intramyocardial signalling and matrix remodelling and capillary rarefaction, biomarkers reflecting these processes could aid in diagnostic and pathophysiological stratification in HFpEF patients. Recently, several biomarkers were identified, which appeared to be promising diagnostic and prognostic tools in patients with HFpEF (Table 2; [48]).
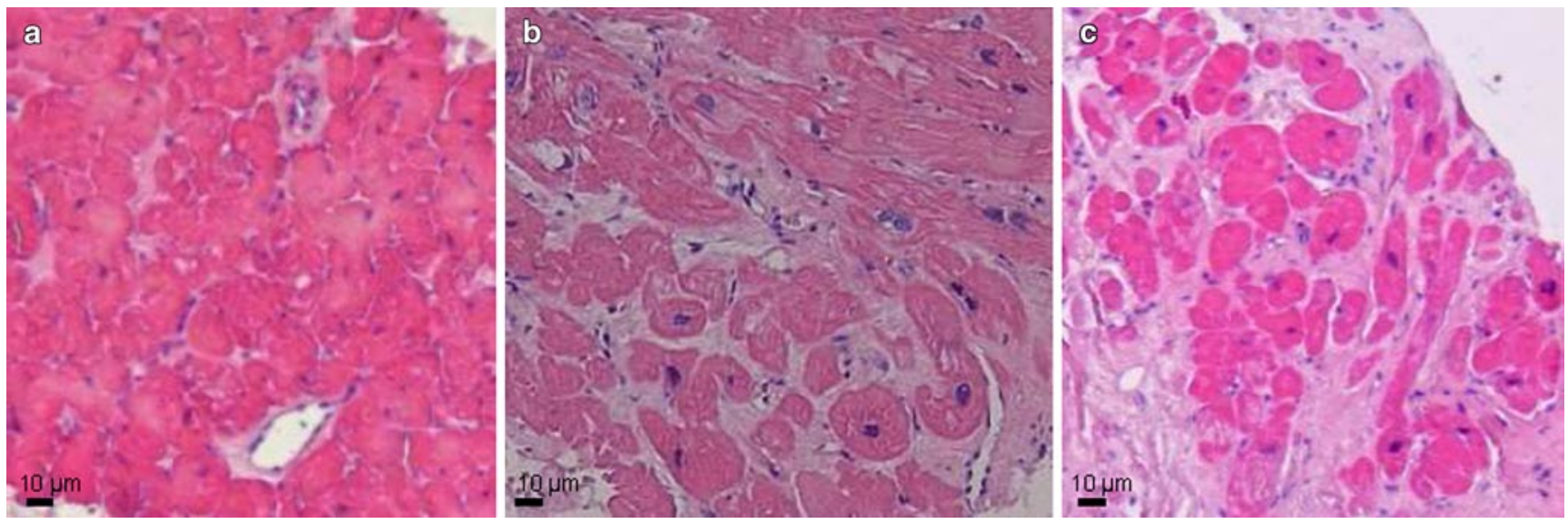

Fig. 5 Distinct stages of structural myocardial disease in HFpEF. a-c, histological images of LV myocardium from HFpEF patients, demonstrating minor (a), moderate (b) and severe (c) interstitial fibrosis 
Table 2 Potential biomarkers for identification of underlying disease processes in HFpEF

\begin{tabular}{|c|c|}
\hline $\begin{array}{l}\text { Pathophysiological } \\
\text { process }\end{array}$ & Biomarkers \\
\hline Inflammation & $\begin{array}{l}\text { CRP, IL-6, IL-8, IL-10, TNF- } \alpha \text {, Pen- } \\
\text { traxin-3, Galectin-3, MCP-1, GDF-15, } \\
\text { Soluble ST2 }\end{array}$ \\
\hline $\begin{array}{l}\text { Extracellular matrix } \\
\text { remodelling }\end{array}$ & $\begin{array}{l}\text { MMPs, TIMPs, Collagen propeptides } \\
\text { (PICP, PINP, PIIINP, CITP, Galectin-3 }\end{array}$ \\
\hline Myocyte stress & $\begin{array}{l}\text { (NT-pro)BNP, ANP, (nt-proCNP), } \\
\text { GDF-15 }\end{array}$ \\
\hline Myocyte injury/apoptosis & Troponins, Galectin-3 \\
\hline Endothelial dysfunction & $\begin{array}{l}\text { E-selectin, P-selectin, VCAM-1, ICAM- } \\
\text { 1, (NT-proCNP), cGMP }\end{array}$ \\
\hline Oxidative stress & Nitrotyrosine \\
\hline Renal dysfunction & Cystatin-c, microalbuminuria \\
\hline Miscellaneous & $\begin{array}{l}\text { Homocysteine, advanced glycation end } \\
\text { products }\end{array}$ \\
\hline
\end{tabular}

CRP C-reactive protein, $I L-6$ interleukin $6, I L-8$ interleukin $8, I L-10$ interleukin 10, MCP-1 monocyte chemoattractant protein 1, GDF-15 growth differentiation factor $15, M M P s$ matrix metalloproteinases, TIMPs tissue inhibitor of MMPs, PICP C-terminal propeptide of procollagen type 1 , PINP N-terminal propeptide of procollagen type 1, PIIINP N-terminal propeptide of procollagen type III, CITP C-terminal telopeptide of collagen type I, ICAM-1 intercellular adhesion molecule 1 .

\section{Imaging}

Cardiac magnetic resonance imaging with $\mathrm{T} 1$ mapping represents a novel technique, which quantifies the myocardial extracellular volume and that significantly correlates with histological myocardial interstitial fibrosis and LV stiffness [49]. In HFpEF patients, reduced T1 times, indicative of increased interstitial myocardial fibrosis, was associated with worse outcome [50]. Hence, biomarker- and/or imaging-guided quantification of myocardial interstitial fibrosis could aid in selection of HFpEF patients for specific therapeutic strategies.

\section{Conclusion}

HFpEF is a complex and heterogeneous clinical syndrome, which is associated with multiple comorbidities, pathogenic mechanisms and lack of effective treatment. Over the past decade, significant progress has been made in understanding HFpEF pathophysiology and recognising the importance of comorbidities and disease heterogeneity. Importantly, the conceptual framework of HFpEF treatment may need to shift from a 'one fits all' strategy to an individualised approach based on phenotypic patient characterisation and diagnostic and pathophysiological stratification of myocardial disease processes. This could ultimately provide an inroad for improved identification of specific treatment targets and enhance development of 'personalised therapy' for HFpEF patients.
Funding This work was supported by a grant from the European Commission (FP7-Health-2010; MEDIA-261409).

Conflict of interest None declared.

Open Access This article is distributed under the terms of the Creative Commons Attribution 4.0 International License (http://creativecommons.org/licenses/by/4.0/), which permits unrestricted use, distribution, and reproduction in any medium, provided you give appropriate credit to the original author(s) and the source, provide a link to the Creative Commons license, and indicate if changes were made.

\section{References}

1. Steinberg BA, Zhao X, Heidenreich PA, Get With the Guidelines Scientific Advisory Committee and Investigators, et al. Trends in patients hospitalized with heart failure and preserved left ventricular ejection fraction: prevalence, therapies, and outcomes. Circulation. 2012;126:65-75.

2. Paulus WJ, Tschöpe C, Sanderson JE, et al. How to diagnose diastolic heart failure: a consensus statement on the diagnosis of heart failure with normal left ventricular ejection fraction by the Heart Failure and Echocardiography Associations of the European Society of Cardiology. Eur Heart J. 2007;28:2539-50.

3. Yusuf S, Pfeffer MA, Swedberg K, for the CHARM Investigators and Committees, et al. Effects of candesartan in patients with chronic heart failure and preserved left-ventricular ejection fraction: the CHARM-Preserved Trial. Lancet. 2003;362:777-81.

4. Cleland JG, Tendera M, Adamus J, PEP-CHF Investigators, et al. The perindopril in elderly people with chronic heart failure (PEPCHF) study. Eur Heart J. 2006;27:2338-45.

5. Massie BM, Carson PE, McMurray JJ, I-PRESERVE Investigators, et al. Irbesartan in patients with heart failure and preserved ejection fraction. N Engl J Med. 2008;359:2456-67.

6. Redfield MM, Chen HH, Borlaug BA, RELAX Trial, et al. Effect of phosphodiesterase- 5 inhibition on exercise capacity and clinical status in heart failure with preserved ejection fraction: a randomized clinical trial. JAMA. 2013;309:1268-77.

7. Pitt B, Pfeffer MA, Assmann SF, TOPCAT Investigators, et al. Spironolactone for heart failure with preserved ejection fraction. $\mathrm{N}$ Engl J Med. 2014;370:1383-92.

8. Senni M, Paulus WJ, Gavazzi A, et al. New strategies for heart failure with preserved ejection fraction: the importance of targeted therapies for heart failure phenotypes. Eur Heart J. 2014;35:2797-811.

9. Zile MR, Baicu CF, Gaasch WH. Diastolic heart failure-abnormalities in active relaxation and passive stiffness of the left ventricle. N Engl J Med. 2004;350:1953-59.

10. Westermann D, Kasner M, Steendijk P, et al. Role of left ventricular stiffness in heart failure with normal ejection fraction. Circulation. 2008; 117:2051-60.

11. Borbely A, Velden J van der, Papp Z, et al. Cardiomyocyte stiffness in diastolic heart failure. Circulation. 2005;111:774-81.

12. Heerebeek L van, Borbely A, Niessen HWM, et al. Myocardial structure and function differ in systolic and diastolic heart failure. Circulation. 2006;113:1966-73.

13. Heerebeek L van, Hamdani N, Handoko ML, et al. Diastolic stiffness of the failing diabetic heart: importance of fibrosis, advanced glycation endproducts, and myocyte resting tension. Circulation. 2008;117:43-51.

14. Borbely A, Falcao-Pires I, Heerebeek van, et al. Hypophosphorylation of the stiff N2B titin isoform raises cardiomyocyte resting tension in failing human myocardium. Circ Res. 2009;104:780-6. 
15. Heerebeek L van, Hamdani N, Falcao-Pires I, et al. Low myocardial protein kinase $\mathrm{G}$ activity in heart failure with preserved ejection fraction. Circulation. 2012;126:830-9.

16. Westermann D, Lindner D, Kasner M, et al. Cardiac inflammation contributes to changes in the extracellular matrix in patients with heart failure and normal ejection fraction. Circ Heart Fail. 2011;4:44-52.

17. Kasner M, Westermann D, Lopez B, et al. Diastolic tissue Doppler indexes correlate with the degree of collagen expression and crosslinking in heart failure and normal ejection fraction. J Am Coll Cardiol. 2011;57:977-85.

18. Mohammed SF, Hussain S, Mirzoyev SA, et al. Coronary microvascular rarefaction and myocardial fibrosis in heart failure with preserved ejection fraction. Circulation. 2015;131:550-9.

19. Linke WA, Hamdani N. Gigantic business: titin properties and function through thick and thin. Circ Res. 2014;114:1052-68.

20. Takimoto E. Cyclic GMP-dependent signaling in cardiac myocytes. Circ J. 2012;76:1819-25.

21. Madamanchi C, Alhosaini H, Sumida A, et al. Obesity and natriuretic peptides, BNP and NT-proBNP: mechanisms and diagnostic implications for heart failure. Int J Cardiol. 2014;176:611-7.

22. Chirinos JA, Segers P, Gupta AK, et al. Time-varying myocardial stress and systolic pressure-stress relationship: role in myocardialarterial coupling in hypertension. Circulation. 2009;119:2798-807.

23. Lee DI, Zhu G, Sasaki T, et al. Phosphodiesterase 9A controls nitric-oxide-independent cGMP and hypertrophic heart disease. Nature. 2015;519:472-6.

24. Yancy CW, Lopatin M, Stevenson LW, et al. for the ADHERE Scientific Advisory Committee and Investigators. Clinical presentation, management, and in-hospital outcomes of patients admitted with acute decompensated heart failure with preserved systolic function. A Report from the Acute Decompensated Heart Failure National Registry (ADHERE). J Am Coll Cardiol. 2006;47:76-84.

25. Fonorow GC, Stough WG, Abraham WT, OPTIMIZE-HF Investigators and Hospitals, et al. Characteristics, treatments and outcomes of patients with preserved systolic function hospitalized for heart failure: a report from the OPTIMIZE-HF Registry. J Am Coll Cardiol. 2007;50:768-77.

26. Ather S, Chan W, Bozkurt B, et al. Impact of noncardiac comorbidities on morbidity and mortality in a predominantly male population with heart failure and preserved versus reduced ejection fraction. J Am Coll Cardiol. 2012;59:998-1005.

27. Akiyama E, Sugiyama S, Matsuzawa Y, et al. Incremental prognostic significance of peripheral endothelial dysfunction in patients with heart failure with normal left ventricular ejection fraction. J Am Coll Cardiol. 2012;60:1778-86.

28. Paulus WJ, Tschöpe C. A novel paradigm for heart failure with preserved ejection fraction: comorbidities drive myocardial dysfunction and remodeling through coronary microvascular endothelial inflammation. J Am Coll Cardiol. 2013;62:263-71.

29. Goligorsky MS. Microvascular rarefaction: the decline and fall of blood vessels. Organogenesis. 2010;6:1-10.

30. Hoenig MR, Bianchi C, Rosenzweig A, et al. The cardiac microvasculature in hypertension, cardiac hypertrophy and diastolic heart failure. Curr Vasc Pharmacol. 2008;6:292-300.

31. Campbell DJ, Somaratne JB, Prior DL, et al. Obesity is associated with lower coronary microvascular density. PLoS One. 2013;8:e81798.

32. Borlaug BA, Lam CS, Roger VL, et al. Contractility and ventricular systolic stiffening in hypertensive heart disease insights into the pathogenesis of heart failure with preserved ejection fraction. $\mathrm{J}$ Am Coll Cardiol. 2009;54:410-8.

33. Phan TT, Abozguia K, Nallur Shivu G, et al. Heart failure with preserved ejection fraction is characterized by dynamic impairment of active relaxation and contraction of the left ventricle on exercise and associated with myocardial energy deficiency. J Am Coll Cardiol. 2009;54:402-9.
34. Kawaguchi M, Hay I, Tetics B, et al. Combined ventricular systolic and arterial stiffening in patients with heart failure and preserved ejection fraction: implications for systolic and diastolic reserve limitations. Circulation. 2003;107:714-20.

35. Borlaug BA, Olson TP, Lam CS, et al. Global cardiovascular reserve dysfunction in heart failure with preserved ejection fraction. J Am Coll Cardiol. 2010;56:845-54.

36. Lam CS, Roger VL, Rodeheffer RJ, et al. Pulmonary hypertension in heart failure with preserved ejection fraction: a communitybased study. J Am Coll Cardiol. 2009;53:1119-26.

37. Melenovsky V, Hwang SJ, Lin G, et al. Right heart dysfunction in heart failure with preserved ejection fraction. Eur Heart J. 2014;35:3452-62.

38. Burke MA, Katz DH, Beussink L, et al. Prognostic importance of pathophysiologic markers in patients with heart failure and preserved ejection fraction. Circ Heart Fail. 2014;7:288-99.

39. Borlaug BA, Kass DA. Ventricular-vascular interaction in heart failure. Heart Fail Clin. 2008;4:23-36.

40. Marti CN, Gheorghiade M, Kalogeropoulos AP, et al. Endothelial dysfunction, arterial stiffness, and heart failure. J Am Coll Cardiol. 2012;60:1455-69.

41. Borlaug BA, Melenovsky V, Russell SD, et al. Impaired chronotropic and vasodilator reserves limit exercise capacity in patients with heart failure and a preserved ejection fraction. Circulation. 2006;114:2138-47.

42. Shah SJ, Katz DH, Selvaraj S, et al. Phenomapping for novel classification of heart failure with preserved ejection fraction. Circulation. 2015;131:269-79.

43. Guazzi M, Vicenzi M, Arena R, et al. Pulmonary hypertension in heart failure with preserved ejection fraction: a target of phosphodiesterase-5 inhibition in a 1-year study. Circulation. 2011;124:164-74.

44. Melenovsky V, Borlaug BA, Rosen B, et al. Cardiovascular features of heart failure with preserved ejection fraction versus nonfailing hypertensive left ventricular hypertrophy in the urban Baltimore community: the role of atrial remodeling/dysfunction. J Am Coll Cardiol. 2007;49:198-207.

45. Anand IS, Rector TS, Cleland JG, et al. Prognostic value of baseline plasma amino-terminal pro-brain natriuretic peptide and its interactions with irbesartan treatment effects in patients with heart failure and preserved ejection fraction: findings from the I-PRESERVE trial. Circ Heart Fail. 2011;4:569-77.

46. Pfeffer MA, Clagget B, Assmann SF, et al. Regional variation in patients and outcomes in the Treatment of Preserved Cardiac Function Heart Failure With an Aldosterone Antagonist (TOPCAT) trial. Circulation. 2015;131:34-42.

47. Zakeri R, Borlaug BA, McNulty SE, et al. Impact of atrial fibrillation on exercise capacity in heart failure with preserved ejection fraction: a RELAX trial ancillary study. Circ Heart Fail. 2014;7:123-30.

48. Cheng JM, Akkerhuis KM, Battes LC, et al. Biomarkers for heart failure with normal ejection fraction: a systematic review. Eur $\mathrm{J}$ Heart Fail. 2013;15:1350-62.

49. Iles L, Pfluger H, Phrommintikul A, et al. Evaluation of diffuse myocardial fibrosis in heart failure with cardiac magnetic resonance contrast-enhanced T1 mapping. J Am Coll Cardiol. 2008;52:1574-80.

50. Mascherbauer J, Marzluf BA, Tufaro C, et al. Cardiac magnetic resonance postcontrast $\mathrm{T} 1$ time is associated with outcome in patients with heart failure and preserved ejection fraction. Circ Cardiovasc Imaging. 2013;6:1056-65. 